\title{
SUSTAINABLE GROWTH RATE OF AGRICULTURAL AND FOOD ENTERPRISES IN SERBIA ${ }^{1}$
}

\author{
Zoran Jović², Kosana Vićentijevićc ${ }^{3}$, Nataša Glišovićc ${ }^{4}$
}

\begin{abstract}
Summary
Many profitable businesses can get bankrupt if they grow too slowly or too fast. In order to assess whether a company is growing too slowly or too fast, it is necessary to establish a sustainable growth rate, that represents the maximum growth rate which the company can achieve without additional debt growing. The aim of this study is to determine the sustainable growth rate for the enterprises of the agricultural and food sector in Serbia in 2012 and 2013 and to determine whether there are differences between these two interconnected sectors. The construction sector which is not reproductively connected with agriculture and food ones, was introduced in the study as a control sector. The aim is also to establish a real sustainable growth rate of observed sectors in Serbia taking into consideration the inflation rate which in Serbia has visible fluctuations for the observed years. Real sustainable growth rate serves as a landmark for drawing conclusions about the development potential of these branches and as a conclusion of which internal or external incentives are needed to further increase sustainable growth rate, namely potential for growth. Limitation of the work scope focused research on identifying sustainable growth rate and mutual comparison of observed sectors and provided a basis for further research in the direction of the comparative analysis within the agricultural sector, as well as for determining agricultural activities with the greatest growth potential, expressed through the sustainable growth rate.
\end{abstract}

Keywords: sustainable growth rate, the agricultural sector, food sector, dividend policy

JEL: $Q 14, O 47, D$ 92, $C 10$

1 Acknowledgement: The work presented here was supported by the Serbian Ministry of Education and Science (project No. III44006)

2 Zoran Jović, Ph.D., Associate Professor, University Singidunum, Faculty of Business Valjevo, Železnička Street no. 5, 14000 Valjevo, Serbia, Phone: +381 14292 610, E-mail: zjovic@singidunum.ac.rs

3 Kosana Vićentijević, Ph.D., Associate Professor, University Singidunum Belgrade, Danijelova Street no. 32, 11000 Beograd, Serbia, Phone: +381 113093 209, E-mail: kvicentijevic@singidunum.ac.rs

4 Nataša Glišović, M.A., Mathematical Institute of the Serbian Academy of Arts and Sciences (SANU), Kneza Mihaila Street no. 36, 11000 Belgrade, Serbia, Phone: + 381641271879 , E-mail: natasaglisovic@gmail.com 


\section{Introduction}

The term sustainable growth rate of the company means the maximum growth rate which the company can achieve without new issues of equity and without additional debt growing. If the company is growing at a rate that is lower than sustainable, as a rule it can lead to stagnation of the company, loss of competitive position, and in the extreme case to bankruptcy of the company. On the other hand, if the company is growing at a rate that is higher than the sustainable growth rate, the company may be imposed in financial troubles, lack of liquidity, and ultimately, it may also lead to the bankruptcy of the company. In order to be able to finance rapid growth, a company has at its disposal multiple options that can have internal or external orientation. Internal options may be aimed at increasing production efficiency, improving total asset turnover ratios and all of its individual parts, and external options may be facing increasing debt or recapitalization through a new issue of shares.

The subject of this paper is to analyze the sustainable growth rate of agricultural and food enterprises in Serbia. From the theoretical point of defining research subject, the methodology for determining sustainable growth rate is shown, as well as the research results of some foreign authors.

In accordance with the modern techniques of the methodology of scientific research, conducted research has both theoretical and empirical character. The theoretical aspect of the analysis is supported by knowledge based on foreign expert, scientific sources and findings of other authors who have dealt with this issue. Empirical aspect of the analysis is supported by the current study of the authors with statistical representations performed on basis of official data.

Based on the specificity and complexity of the studied topic in order to satisfy the basic methodological requirements of objectivity, reliability, generality and systematics, in this research the basic scientific methods of analysis and synthesis, inductive and deductive methods, description and method of comparison, as well as general research methods such as comparative-historical method, statistical method and hypothetical - deductive method were used. Among the methods of data collection the method of content analysis of documents was used.

The study focuses on the enterprises in Serbia and their comparative analysis. We analyzed 20 agricultural enterprises, 20 enterprises of the food industry and 20 construction enterprises. Attention is focused on the agricultural sector and enterprises of this sector, because thanks to its natural predispositions Serbia has great development opportunities in the field of agricultural production. Since the agricultural production is base for development of the food industry, hence this industry has great development potential in mutual coupling between agriculture and food industries. That fact identified a particular interest of authors to analyze sustainable growth rates of these two industries. Construction enterprises are the third group of enterprises in which this research determined sustainable growth rate. For the construction industry it could be said that it is reproductively independent from agricultural production and 
food industry, which are mutually reproductively linked. Therefore, the construction enterprises were selected as a control sector independent from the agricultural and food enterprises sectors.

The study is a comparative analysis of the sustainable growth rate of agricultural, food and construction enterprises, in 2012 and 2013. According to the authors' knowledge in Serbia there was not any research done on the topic of establishing a sustainable growth rate of a company in this way and for this period, hence the aim of this paper is to show the methodology for calculating the sustainable growth rate, and based on it calculate the growth rate for 60 enterprises from Serbia in 2012 and 2013, from agriculture, food and construction sectors. It also seeks to determine whether or not there are significant differences between them in the amount of sustainable growth rates in 2013 compared to the year 2012. In addition, we analyze the relationship between the sustainable growth rate and the inflation rate in order to determine the real potential for growth in these sectors.

Bearing in mind the above mentioned, the report is based on the following proposed hypothesis:

H1: There is no significant difference between the sustainable growth rate of agricultural, food and construction industry enterprises.

$\mathrm{H} 2$ : There is no statistically significant difference in the level of sustainable growth rates of agricultural, food and construction enterprises in 2012.

H3: There is no statistically significant difference in the level of sustainable growth rates of agricultural, food and construction enterprises in 2013.

H4: There is no statistically significant difference in the level of sustainable growth rates of agricultural, food and construction enterprises, as well as in the level of sustainable growth rates of all the enterprises in the taken sample in 2013 compared to 2012.

H5: There is no statistically significant difference between the average sustainable growth rate and inflation rate in 2012 .

H6: There is no statistically significant difference between the average sustainable growth rate and inflation rate in 2013.

\section{Literature rewiew}

By determining the growth rate that the company can afford stating that unbridled growth could be contrary to financial policy of the company (Higgins, RC, 1977), it can be concluded that in terms of maintaining the desired capital structure and targeted ratio of dividend payments, without issuing new shares, sustainable growth rate represents percentage in increased sales in accordance with the financial policy of the company. The increase in sales over the sustainable growth rate creates for the company financial problems by requiring new loans to finance rapid growth. Since increase in sales may be a result of the increased volume of products or increased prices of the product, 
the impact of inflation on a sustainable growth rate is considered as well (Higgins, $\mathrm{RC}, 1981)$. One of the important tasks for both small business owners, and managers of large corporations is to establish a sustainable growth rate, namely the maximum growth rate that the company can sustain without increasing its financial leverage (Brealey, Myers, 2003). The sustainable growth rate is also defined as the growth rate of dividends (and profit) that can be held for a given level of return of capital, under the assumption that the constant capital structure is kept, and that additional share issues are not issued (Pinto et al., 2010; Gordon, 1959; Fuller, Hsia, 1984). In determining the sustainable growth rate one should start from the real possibilities of a company and from situation at the financial market and then to determine, based on the target operating ratio, debt and dividend payments, the maximum increase percentage in sales revenue, with an emphasis that any increase in assets must be equal to the increase in liabilities and equity capital through increase in retained earnings (Van Horne, Wachowicz, 2007). By implementing moderate mathematical modification of Higginson's model for calculating the leverage ratio by taking the same cut-off date for total assets and shareholders' equity at the beginning of the period, instaed at the end of the period as Higginson did, the accuracy of Higginson's model was improved (Ashta, 2008). It was investigated which model is more suitable for determining the sustainable growth rate by comparing Higginson's and Van Horn's models (Fonseca et al., 2012). It was concluded that in determining the sustainable growth rate of profitable enterprises Higginson's model gives higher sustainable growth rate than Van Horn's model. The same study revealed that in companies with high leverage, Van Horn's model provides a higher sustainable growth rate than Higginson's model, but it was concluded that the differences in the models are not significant and that both models give satisfactory results in the study.

The company's ability to fund its future development was studied on the example of the sustainable growth rate of an airline (Chang, YC, 2012). Analyzed actual and sustainable Higgins growth rate showed large fluctuations until the moment when studied airline merged with another airline. After the merger of these two airlines, a gradual increase and convergence of actual and sustainable growth rates were recorded. The relation between actual and sustainable growth rates was investigated in the case of US gas industry in the period from 1970 to 1990 (Clouse, McFaddin, 1994). Research showed that the potential for sustainable growth in US gas industry is in the future shares issuing, operational improvements and change of financial goals. It was concluded that the model of sustainable growth represents an efficient tool for financial planning and directing the business policy toward growth stimulation in this industry. The relation between the current and sustainable growth rates of small and mediumsized enterprses in Canada in the period 2000-2010 was investigated on the example of several economic sectors: primary, construction, manufacturing, wholesale trade, retail trade, science and technology, accommodation and food services and other services (Seens, 2013). The results support the hypothesis that the sector of the firm does affect the firm's sustainable growth rate. The differences in growth rates between sectors were 
found to be statistically significant. The sustainable growth rate of the construction and service sectors were significantly higher than in the primary and manufacturing sectors. The manner in which the two car companies in India, Tata Motors and Maruti Suzuki used internally generated funds to improve their operational and financial performance, achieve progress and use the rapidly growing car industry, was shown by the case study on the measures' improving to achieve sustainable growth rates of these two companies (Virani, 2013). Higginson's model of sustainable growth model that optimizes the sustainable growth rate and ratio of dividend payouts was extended by the study of the relation between investment decisions on the optimal growth and dividend policy (Chen et al., 2013). This research has shown that the covariance between profitability and growth rate is one of the determinants of dividend policy and that the interaction between the risk of profitability and growth rate can affect the permanent ceasing of dividend payouts of companies.

\section{The methodology of determining the sustainable growth rate}

Calculation of the sustainable growth rate was done on the database of 60 enterprises from the Republic of Serbia (20 enterprises from each of the three sectors - agriculture, food and construction) whose shares are traded on the Belgrade Stock Exchange. The stratified sampling method was used in the process of forming the sample, by forming three strata, which were comprized of all companies whose shares are traded on the BSE, and which, at the same time were part of the agriculture, food or construction sectors. Prime sample of 20 companies was elected for each of the three observed strata. The union of such three random, simple samples constitute stratified sample on which basis the subject research is made. Data about the inflation rate as measured by the consumer price index for 2012 and 2013 are taken from the website of the Ministry of Finance of the Republic of Serbia (Ministarstvo finansija, 2014).

For determinating sustainable growth rate the next formula can be used:

$\mathrm{g}=\mathrm{b} \times \mathrm{ROE}$

where:

$\mathrm{g}$ - is sustainable growth rate

$\mathrm{b}$ - is retention rate (plowback ratio)

ROE - return on equity

Retentio rate (b) is calculated using the next formula:

$\mathrm{b}=1$ - dividend payout ratio $=($ net income - dividends $) /$ net income

By applying DuPont analysis, the rate of return on equity can be broken down into the following components: profit margin, total assets turnover ratio and equity multiplier (Stowe, J., 2000):

$\mathrm{ROE}=$ profit margin $\mathrm{x}$ total assets turnover ratio $\mathrm{x}$ equity multiplier 


$$
\text { ROE }=\frac{\text { Net income }}{\text { Shareholders equity }}=\frac{\text { Net income }}{\text { Sales revenue }} \times \frac{\text { Sales revenue }}{\text { Total assets }} \times \frac{\text { Total assets }}{\text { Shareholdes'equity }}
$$

On this basis, the initial formula for calculating the sustainable growth rate can be transformed into (Stowe, J., 2000):

$$
\mathrm{g}=\frac{\text { Net income }- \text { Dividends }}{\text { Net income }} \times \frac{\text { Net income }}{\text { Sales revenue }} \times \frac{\text { Sales revenue }}{\text { Total assets }} \times \frac{\text { Total assets }}{\text { Shareholdes'equity }}
$$

The paper uses this expanded form of the formula for calculating the sustainable growth rate, since it may provide useful information in analyzing the factors that affect its height.

To test the hypothesis 2 and 3, ie. to determine the existence of statistically significant differences among the sustainable growth rate of enterprises in the agricultural, food and construction sectors in 2012 and 2013 Kruskal-Wallis's test was used.

To test the hypothesis 4 , ie. to determine the existence of statistically significant differences in the overall level of sustainable growth rates in 2013 compared to 2012, the Wilcoxon Signed Rank test was used.

Testing of hypotheses 5 and 6 in order to determine whether there is a statistically significant difference between the average sustainable growth rate of sampled companies and the inflation rate in 2012 and 2013 was based on One-Sample T test.

For all calculations software package IBM SPSS Statistics 19 was used.

\section{Establishing sustainable growth rate on agricultural and food enterprises in Serbia}

Based on previouly established methodology sustainable growth rates $(\mathrm{g})$ for 20 sampled agricultural companies in 2012 and 2013 were calculated. The results are shown in Tables 1 and 2.

Table 1. Sustainable growth rates of sampled agricultural enterprises in 2012

\begin{tabular}{|l|r|r|r|r|r|r|}
\hline \multirow{2}{*}{ Enterprise } & \multicolumn{1}{c|}{ b } & \multicolumn{1}{c|}{ ROE } & $\begin{array}{c}\text { profit } \\
\text { margin }\end{array}$ & $\begin{array}{c}\text { total assets } \\
\text { turnover } \\
\text { ratio }\end{array}$ & $\begin{array}{c}\text { equity } \\
\text { multiplier }\end{array}$ & g \\
\cline { 2 - 7 } & $\mathbf{( 1 )}$ & $(\mathbf{2})=(\mathbf{3} * \mathbf{4} * \mathbf{5})$ & \multicolumn{1}{c|}{$\mathbf{( 3 )}$} & $\mathbf{( 4 )}$ & $\mathbf{( 5 )}$ & $\begin{array}{c}(\mathbf{6})= \\
(\mathbf{1} * \mathbf{2})\end{array}$ \\
\hline Agrobačka, Bačka Topola & 1.00 & 1.23 & 2.01 & 0.57 & 1.05 & 1.23 \\
\hline Bezdan, Bezdan & 1.00 & 13.49 & 39.05 & 0.34 & 1.02 & 13.49 \\
\hline Borac, Šurjan & 0.81 & 24.73 & 9.31 & 1.48 & 1.80 & 20.03 \\
\hline
\end{tabular}




\begin{tabular}{|c|c|c|c|c|c|c|}
\hline \multirow[t]{2}{*}{ Enterprise } & b & ROE & $\begin{array}{c}\text { profit } \\
\text { margin }\end{array}$ & $\begin{array}{c}\text { total assets } \\
\text { turnover } \\
\text { ratio }\end{array}$ & $\begin{array}{c}\text { equity } \\
\text { multiplier }\end{array}$ & g \\
\hline & (1) & $(2)=(3 * 4 * 5)$ & (3) & (4) & (5) & $\begin{array}{l}(6)= \\
(1 * 2)\end{array}$ \\
\hline Graničar, Konak & 0.75 & 28.37 & 18.99 & 1.25 & 1.20 & 21.28 \\
\hline Hajdučica, Hajdučica & 1.00 & 2.05 & 1.06 & 1.13 & 1.72 & 2.05 \\
\hline Kačarevo, Kačarevo & 1.00 & 12.70 & 11.32 & 0.69 & 1.63 & 12.70 \\
\hline Omoljica, Omoljica & 1.00 & 9.62 & 19.48 & 0.40 & 1.24 & 9.62 \\
\hline Lučić, Prigrevica & 1.00 & 1.24 & 4.54 & 0.16 & 1.72 & 1.24 \\
\hline Napredak, Stara Pazova & 1.00 & 2.13 & 4.23 & 0.37 & 1.37 & 2.13 \\
\hline $\begin{array}{l}\text { Dragan Marković, } \\
\text { Obrenovac }\end{array}$ & 1.00 & 0.77 & 0.80 & 0.33 & 2.89 & 0.77 \\
\hline PKB Korporacija, Beograd & 1.00 & 1.39 & 6.28 & 0.14 & 1.62 & 1.39 \\
\hline Orahovo, Novo Orahovo & 1.00 & 5.89 & 2.39 & 1.28 & 1.94 & 5.89 \\
\hline PD Zaječar, Zaječar & 1.00 & 17.26 & 33.82 & 0.44 & 1.16 & 17.26 \\
\hline Pionir, Srbobran & 0.38 & 27.39 & 25.53 & 0.95 & 1.12 & 10.41 \\
\hline Pobeda, Pobeda & 1.00 & 0.63 & 0.48 & 0.71 & 1.84 & 0.63 \\
\hline Podunavlje, Čelarevo & 1.00 & 6.12 & 14.87 & 0.15 & 2.77 & 6.12 \\
\hline Sava Kovačević, Vrbas & 0.72 & 11.16 & 11.73 & 0.38 & 2.48 & 8.04 \\
\hline Sloga, Kać & 1.00 & 6.23 & 26.33 & 0.19 & 1.24 & 6.23 \\
\hline $\begin{array}{l}\text { Vino Kalem, Velika } \\
\text { drenova }\end{array}$ & 1.00 & 45.97 & 16.56 & 0.57 & 4.85 & 45.97 \\
\hline Vojvodina, Sombor & 1.00 & 1.64 & 2.79 & 0.30 & 1.94 & 1.64 \\
\hline
\end{tabular}

Source: Representation by the authors based on data from $\underline{w w w . b e l e x . r s}$

Table 2. Sustainable growth rates of sampled agricultural enterprises in 2013

\begin{tabular}{|c|c|c|c|c|c|c|}
\hline \multirow[t]{2}{*}{ Enterprise } & b & ROE & $\begin{array}{l}\text { profit } \\
\text { margin }\end{array}$ & $\begin{array}{c}\text { total assets } \\
\text { turnover } \\
\text { ratio } \\
\end{array}$ & $\begin{array}{l}\text { equity } \\
\text { multiplier }\end{array}$ & $\mathbf{g}$ \\
\hline & (1) & $\begin{array}{c}(2)= \\
(3 * 4 * 5)\end{array}$ & (3) & (4) & (5) & $\begin{array}{l}(6)= \\
(1 * 2)\end{array}$ \\
\hline $\begin{array}{l}\text { Agrobačka, Bačka } \\
\text { Topola }\end{array}$ & 0.999 & 0.95 & 1.63 & 0.47 & 1.22 & 0.95 \\
\hline Bezdan, Bezdan & 1.00 & 10.40 & 25.06 & 0.40 & 1.05 & 10.40 \\
\hline Borac, Šurjan & 0.76 & 15.05 & 6.39 & 1.38 & 1.71 & 11.44 \\
\hline Graničar, Konak & -3.32 & 4.44 & 3.29 & 1.15 & 1.17 & -14.74 \\
\hline Hajdučica, Hajdučica & 1.00 & 0.46 & 0.31 & 0.86 & 1.74 & 0.46 \\
\hline Kačarevo, Kačarevo & 1.00 & 7.35 & 9.32 & 0.52 & 1.51 & 7.35 \\
\hline Omoljica, Omoljica & 1.00 & 0.27 & 2.47 & 0.10 & 1.06 & 0.27 \\
\hline Lučić, Prigrevica & 1.00 & 1.52 & 5.41 & 0.17 & 1.63 & 1.52 \\
\hline Napredak, Stara Pazova & 1.00 & 1.49 & 3.20 & 0.35 & 1.33 & 1.49 \\
\hline $\begin{array}{l}\text { Dragan Marković, } \\
\text { Obrenovac }\end{array}$ & 1.00 & 0.77 & 1.28 & 0.29 & 2.11 & 0.77 \\
\hline
\end{tabular}




\begin{tabular}{|c|c|c|c|c|c|c|}
\hline \multirow[t]{2}{*}{ Enterprise } & $\mathbf{b}$ & ROE & $\begin{array}{c}\text { profit } \\
\text { margin }\end{array}$ & $\begin{array}{c}\text { total assets } \\
\text { turnover } \\
\text { ratio }\end{array}$ & $\begin{array}{l}\text { equity } \\
\text { multiplier }\end{array}$ & g \\
\hline & (1) & $\begin{array}{c}(2)= \\
(3 * 4 * 5)\end{array}$ & (3) & (4) & (5) & $\begin{array}{l}(6)= \\
(1 * 2)\end{array}$ \\
\hline $\begin{array}{l}\text { PKB Korporacija, } \\
\text { Beograd }\end{array}$ & 1.00 & 9.12 & 46.13 & 0.14 & 1.43 & 9.12 \\
\hline $\begin{array}{l}\text { Orahovo, Novo } \\
\text { Orahovo }\end{array}$ & 1.00 & 17.01 & 8.34 & 1.09 & 1.85 & 17.01 \\
\hline PD Zaječar, Zaječar & 1.00 & 3.91 & 6.18 & 0.55 & 1.15 & 3.91 \\
\hline Pionir, Srbobran & 0.16 & 21.80 & 23.89 & 0.78 & 1.17 & 3.49 \\
\hline Pobeda, Pobeda & 1.00 & 1.13 & 0.75 & 0.83 & 1.80 & 1.13 \\
\hline Podunavlje, Čelarevo & 1.00 & 3.38 & 7.80 & 0.15 & 2.85 & 3.38 \\
\hline Sava Kovačević, Vrbas & 0.80 & 21.72 & 29.84 & 0.39 & 1.85 & 17.38 \\
\hline Sloga, Kać & 1.00 & 1.62 & 6.34 & 0.21 & 1.20 & 1.62 \\
\hline $\begin{array}{l}\text { Vino Kalem, Velika } \\
\text { drenova }\end{array}$ & 1.00 & 32.46 & 13.52 & 0.58 & 4.12 & 32.46 \\
\hline Vojvodina, Sombor & 1.00 & 6.09 & 9.94 & 0.37 & 1.64 & 6.09 \\
\hline
\end{tabular}

Source: Representation by the authors based on data from www.belex.rs

It may be noted that there was no change in the policy of dividend payouts - during both years the same four companies paid out part of the profit as dividends to its shareholders, while sixteen companies did not pay dividends to shareholders, i.e. reinvested overall profit. Agricultural companies that payout dividends from net profit and thus reinvest a smaller share of the profit do not have, as might be expected, the lowest sustainable growth rate. The reason is that these companies have high rate on return on equity ROE. The exception is the company Graničar - Konak, which paidout dividends for 2013 from the overall profit in 2013 and from retained earnings from previous years, realizing negative sustainable growth rate of $-14.74 \%$.

The greatest impact on the achieved sustainable growth rate of these agricultural enterprises has profit margin, which is directly transferred to the ROE. The enterprise Bezdan - Bezdan achieved the highest rate of profit margin in 2012 (39.05\%), and in 2013, it did PKB Corporation Belgrade (46.13\%). The ratio of total asset turnover is relatively stable (avarage of 0.59 in 2012; 0.54 in 2013), wherein enterprise Borac, Surjan expressed the highest efficiency of total assets, 1.48 in 2012 and 1.38 in 2013. Own capital multiplier is also relatively stable and it ranges from 1.02 to 4.85 , wherein this indicator for most observed agricultural enterprise is between levels 1 and 2. The highest level of own capital multiplier was recorded by Vino Kalem - Velika Drenova (4.85 in 2012 and 4.12 in 2013), indicating high reliance on borrowed sources of financing.

Sustainable growth rates (g) for the 20 sampled enterprises from food sector in 2012 and 2013 are shown in Tables 3 and 4. 
Table 3. Sustainable growth rates of sampled enterprises of food sector in 2012

\begin{tabular}{|c|c|c|c|c|c|c|}
\hline \multirow[t]{2}{*}{ Enterprise } & b & ROE & $\begin{array}{l}\text { profit } \\
\text { margin }\end{array}$ & $\begin{array}{c}\text { total assets } \\
\text { turnover } \\
\text { ratio } \\
\end{array}$ & $\begin{array}{l}\text { equity } \\
\text { multiplier }\end{array}$ & g \\
\hline & (1) & $\begin{array}{c}(2)= \\
(3 * 4 * 5)\end{array}$ & (3) & (4) & (5) & $\begin{array}{l}(6)= \\
(1 * 2)\end{array}$ \\
\hline Bag, Bačko Gradište & 1.00 & 9.65 & 13.68 & 0.57 & 1.24 & 9.65 \\
\hline Bambi, Požarevac & 0.80 & 25.87 & 13.48 & 0.98 & 1.95 & 20.70 \\
\hline $\begin{array}{l}\text { Banat fabrika ulja, Nova } \\
\text { Crnja }\end{array}$ & -8.04 & 2.73 & 1.12 & 1.12 & 2.17 & -21.95 \\
\hline Čokolend, Paraćin & 0.25 & 7.39 & 2.59 & 1.46 & 1.96 & 1.85 \\
\hline Dijamant, Zrenjanin & 1.00 & 16.43 & 9.61 & 0.78 & 2.20 & 16.34 \\
\hline Oreovica, Oreovica & 1.00 & 1.93 & 0.16 & 4.06 & 2.87 & 1.93 \\
\hline Imlek, Beograd & 0.83 & 25.21 & 13.12 & 0.86 & 2.24 & 20.92 \\
\hline Jabuka, Pančevo & 1.00 & 3.97 & 1.09 & 0.87 & 4.17 & 3.97 \\
\hline $\begin{array}{l}\text { Klanica } 8 \text { oktobar, } \\
\text { Petrovac }\end{array}$ & 1.00 & 2.85 & 0.20 & 2.76 & 5.12 & 2.85 \\
\hline Medela, Vrbas & 1.00 & 5.96 & 9.58 & 0.53 & 1.18 & 5.96 \\
\hline Mlekara, Loznica & 1.00 & 8.73 & 1.32 & 3.60 & 1.84 & 8.73 \\
\hline Mlinprodukt, Ada & 1.00 & 4.16 & 2.27 & 1.01 & 1.81 & 4.16 \\
\hline Neoplanta, Novi Sad & 1.00 & 8.84 & 5.96 & 0.89 & 1.67 & 8.84 \\
\hline Niška mlekara, Niš & 1.00 & 11.69 & 2.98 & 1.53 & 2.56 & 11.69 \\
\hline Rubin, Kruševac & 1.00 & 12.26 & 23.11 & 0.27 & 1.95 & 12.26 \\
\hline Sreten Gudurić, Užice & 0.26 & 12.27 & 2.65 & 2.33 & 1.99 & 3.19 \\
\hline Pekarstvo, Kraljevo & -0.64 & 4.54 & 1.85 & 1.09 & 2.25 & -2.91 \\
\hline Žitobanat, Vršac & 0.52 & 8.17 & 3.17 & 1.60 & 1.62 & 4.25 \\
\hline $\begin{array}{l}\text { Žitopromet - mlin, } \\
\text { Senta }\end{array}$ & 1.00 & 16.35 & 4.62 & 1.21 & 2.92 & 16.35 \\
\hline TE - TO, Senta & 0.54 & 52.67 & 16.80 & 1.35 & 2.33 & 28.44 \\
\hline
\end{tabular}

Source: Representation by the authors based on data from www.belex.rs

Table 4. Sustainable growth rates of sampled enterprises of food sector in 2013

\begin{tabular}{|l|r|r|r|r|r|r|}
\hline \multirow{2}{*}{ Enterprise } & \multicolumn{1}{|c|}{$\mathbf{b}$} & \multicolumn{1}{c|}{ ROE } & \multicolumn{1}{c|}{$\begin{array}{c}\text { profit } \\
\text { margin }\end{array}$} & $\begin{array}{c}\text { total assets } \\
\text { turnover } \\
\text { ratio }\end{array}$ & \multicolumn{1}{c|}{$\begin{array}{c}\text { equity } \\
\text { multiplier }\end{array}$} & g \\
\cline { 2 - 7 } & $\mathbf{( 1 )}$ & $\begin{array}{c}(\mathbf{2})= \\
(\mathbf{3} \mathbf{4} * \mathbf{5})\end{array}$ & \multicolumn{1}{c|}{$\mathbf{( 3 )}$} & \multicolumn{1}{c|}{$\mathbf{( 4 )}$} & $\mathbf{( 5 )}$ & $\begin{array}{r}\mathbf{( 6 )}= \\
(\mathbf{1} * \mathbf{2})\end{array}$ \\
\hline Bag, Bačko Gradište & 1.00 & 4.45 & 5.56 & 0.76 & 1.05 & 4.45 \\
\hline Bambi, Požarevac & 0.80 & 33.69 & 18.86 & 1.10 & 1.63 & 26.95 \\
\hline $\begin{array}{l}\text { Banat fabrika ulja, Nova } \\
\text { Crnja }\end{array}$ & 0.99 & 29.26 & 12.89 & 0.91 & 2.50 & 28.97 \\
\hline Čokolend, Paraćin & 0.16 & 3.75 & 1.24 & 1.59 & 1.91 & 0.60 \\
\hline Dijamant, Zrenjanin & 1.00 & 14.60 & 8.58 & 0.65 & 2.61 & 14.60 \\
\hline Oreovica, Oreovica & 1.00 & 1.61 & 0.15 & 3.23 & 3.32 & 1.61 \\
\hline
\end{tabular}




\begin{tabular}{|l|r|r|r|r|r|r|}
\hline Imlek, Beograd & 0.49 & 22.09 & 10.87 & 0.78 & 2.61 & 10.82 \\
\hline Jabuka, Pančevo & 1.00 & 23.77 & 8.06 & 1.08 & 2.73 & 23.77 \\
\hline $\begin{array}{l}\text { Klanica 8 oktobar, } \\
\text { Petrovac }\end{array}$ & 1.00 & 2.83 & 0.15 & 4.48 & 4.13 & 2.83 \\
\hline Medela, Vrbas & 1.00 & 1.96 & 3.32 & 0.51 & 1.15 & 1.96 \\
\hline Mlekara, Loznica & 1.00 & 7.93 & 1.12 & 3.19 & 2.22 & 7.93 \\
\hline Mlinprodukt, Ada & 1.00 & 6.06 & 2.45 & 1.26 & 1.97 & 6.06 \\
\hline Neoplanta, Novi Sad & 1.00 & 3.61 & 3.53 & 0.78 & 1.31 & 3.61 \\
\hline Niška mlekara, Niš & 1.00 & 14.41 & 3.50 & 1.60 & 2.57 & 14.41 \\
\hline Rubin, Kruševac & 1.00 & 7.71 & 18.20 & 0.26 & 1.62 & 7.71 \\
\hline Sreten Gudurić, Užice & 1.00 & 7.68 & 1.74 & 2.25 & 1.95 & 7.68 \\
\hline Pekarstvo, Kraljevo & 1.00 & 2.14 & 1.24 & 0.80 & 2.16 & 2.14 \\
\hline Žitobanat, Vršac & 0.62 & 6.07 & 2.54 & 1.84 & 1.30 & 3.76 \\
\hline $\begin{array}{l}\text { Žitopromet - mlin, } \\
\text { Senta }\end{array}$ & 1.00 & 15.45 & 4.20 & 1.46 & 2.52 & 15.45 \\
\hline TE - TO, Senta & 0.64 & 18.91 & 8.24 & 0.99 & 2.33 & 12.10 \\
\hline
\end{tabular}

Source: Representation by the authors based on data from www.belex.rs

In 2012 eight companies paidout dividends, and of those eight, two companies (Banat oil factory and Pekarstvo - Kraljevo) paidout higher dividends from the level of the profit on the basis of retained earnings of previous years, which caused negative sustainable growth rate. In 2013, there was a tightening of dividend policy, hence five enterprises paidout dividends from the profit, while none of the enterprises paidout dividends from retained earnings of previous years. It is interesting that the highest sustainable growth rate had companies that paidout the dividend for that year, primarily due to the high ROE levels.

The hihest level of profit margins in 2012 was achieved by Rubin - Kruševac 23.11\% and in 2013 by Bambi - Požarevac 18,86\%. The total asset turnover ratio of food sector is also relatively stable (the average of 1.44 in 2012 and 1.48 in 2013), but it is more than twice the size in the companies of agricultural sector. Own capital multiplier of food enterprises shows relatively stable amounts and it ranges from 1.05 to 5.12, while this indicator for most of these companies is at the level of about 1.05 to 2.5 , ie. on a slightly higher level than in agricultural companies.

It may be noted that the companies of the food sector have somewhat higher rate of return on equity then the companies of the agricultural sector and that agricultural enterprises have significantly higher rates of profit margins then food companies, but lower use efficency of total assets and lower own capital multipliers.

Tables 5 and 6 show sustainable growth rate $(\mathrm{g})$ for the 20 sampled enterprises of the construction sector in 2012 and 2013. 
Table 5. Sustainable growth rates of sampled construction enterprises in 2012

\begin{tabular}{|c|c|c|c|c|c|c|}
\hline \multirow[t]{2}{*}{ Enterprise } & b & ROE & $\begin{array}{l}\text { profit } \\
\text { margin }\end{array}$ & $\begin{array}{l}\text { total assets } \\
\text { turnover } \\
\text { ratio } \\
\end{array}$ & $\begin{array}{l}\text { equity } \\
\text { multiplier }\end{array}$ & g \\
\hline & (1) & $\begin{array}{c}(2)= \\
(3 * 4 * 5)\end{array}$ & (3) & (4) & (5) & $\begin{array}{l}(6)= \\
(1 * 2)\end{array}$ \\
\hline $\begin{array}{l}\text { Energomontaža, } \\
\text { Beograd } \\
\end{array}$ & 1.00 & 1.08 & 0.46 & 1.02 & 2.31 & 1.08 \\
\hline $\begin{array}{l}\text { Energoprojekt oprema, } \\
\text { Beograd }\end{array}$ & 1.00 & 24.43 & 2.37 & 2.72 & 3.79 & 24.43 \\
\hline Erozija, Valjevo & 1.00 & 0.61 & 0.29 & 0.88 & 2.36 & 0.61 \\
\hline $\begin{array}{l}\text { Geosonda - Fundiranje, } \\
\text { Beograd }\end{array}$ & 1.00 & 7.51 & 7.57 & 0.42 & 2.36 & 7.51 \\
\hline $\begin{array}{l}\text { Geosonda } \\
\text { Konsolidacija, Beograd }\end{array}$ & 1.00 & 0.08 & 0.23 & 0.29 & 1.20 & 0.08 \\
\hline Graditelj, Beograd & 1.00 & 0.45 & 0.15 & 1.45 & 2.10 & 0.45 \\
\hline Termika, Beograd & 0.73 & 33.84 & 17.23 & 1.56 & 1.26 & 24.70 \\
\hline $\begin{array}{l}\text { Hidrotehnika } \\
\text {-hidroenergetika, } \\
\text { Beograd } \\
\end{array}$ & 1.00 & 0.72 & 0.35 & 0.72 & 2.85 & 0.72 \\
\hline \begin{tabular}{|l|} 
Zlatibor gradnja, \\
Beograd \\
\end{tabular} & 0.91 & 40.02 & 12.50 & 1.91 & 1.68 & 36.42 \\
\hline Izoprogres, Beograd & 0.91 & 28.58 & 25.98 & 0.74 & 1.48 & 26.01 \\
\hline Jedinstvo, Sevojno & 0.61 & 11.12 & 5.88 & 0.97 & 1.95 & 6.78 \\
\hline Napredak, Pirot & 1.00 & 0.79 & 0.29 & 1.82 & 1.50 & 0.79 \\
\hline $\begin{array}{l}\text { Novi Pazar put, Novi } \\
\text { Pazar }\end{array}$ & 0.63 & 11.42 & 4.99 & 1.04 & 2.19 & 7.19 \\
\hline \begin{tabular}{|l|} 
Planum, Beograd \\
\end{tabular} & 0.00 & 2.17 & 1.05 & 0.88 & 2.36 & 0.00 \\
\hline Putevi, Čačak & 1.00 & 15.08 & 4.93 & 0.88 & 3.49 & 15.08 \\
\hline Putevi, Užice & 1.00 & 1.21 & 0.13 & 0.95 & 9.58 & 1.21 \\
\hline $\begin{array}{l}\text { PZP Požarevac, } \\
\text { Požarevac } \\
\end{array}$ & 1.00 & 3.22 & 1.08 & 1.32 & 2.27 & 3.22 \\
\hline Srbijaput, Beograd & 1.00 & 3.39 & 0.26 & 2.30 & 5.60 & 3.39 \\
\hline Sremput, Ruma & 1.00 & 2.11 & 1.13 & 0.79 & 2.39 & 2.11 \\
\hline Standard, Leskovac & 1.00 & 3.55 & 3.26 & 0.76 & 1.43 & 3.55 \\
\hline
\end{tabular}

Source: Representation by the authors based on data from www.belex.rs

Table 6. Sustainable growth rates of sampled construction enterprises in 2013

\begin{tabular}{|c|c|c|c|c|c|c|}
\hline \multirow{2}{*}{ Enterprise } & b & ROE & $\begin{array}{c}\text { profit } \\
\text { margin }\end{array}$ & $\begin{array}{l}\text { total assets } \\
\text { turnover } \\
\text { ratio } \\
\end{array}$ & $\begin{array}{c}\text { equity } \\
\text { multiplier }\end{array}$ & g \\
\hline & (1) & $\begin{array}{c}(2)= \\
(3 * 4 * 5)\end{array}$ & (3) & (4) & (5) & $(6)=(1 * 2)$ \\
\hline \begin{tabular}{|l|} 
Energomontaža, \\
Beograd \\
\end{tabular} & 1.00 & 1.11 & 0.68 & 0.73 & 2.25 & 1.11 \\
\hline $\begin{array}{l}\text { Energoprojekt oprema, } \\
\text { Beograd }\end{array}$ & 1.00 & 21.06 & 3.66 & 1.68 & 3.42 & 21.06 \\
\hline
\end{tabular}




\begin{tabular}{|c|c|c|c|c|c|c|}
\hline \multirow[t]{2}{*}{ Enterprise } & b & ROE & $\begin{array}{l}\text { profit } \\
\text { margin }\end{array}$ & $\begin{array}{c}\text { total assets } \\
\text { turnover } \\
\text { ratio } \\
\end{array}$ & $\begin{array}{l}\text { equity } \\
\text { multiplier }\end{array}$ & g \\
\hline & (1) & $\begin{array}{c}(2)= \\
(3 * 4 * 5)\end{array}$ & (3) & (4) & (5) & $(6)=(1 * 2)$ \\
\hline Erozija, Valjevo & 1.00 & 9.12 & 5.69 & 0.72 & 2.22 & 9.12 \\
\hline $\begin{array}{l}\text { Geosonda - Fundiranje, } \\
\text { Beograd }\end{array}$ & 1.00 & 0.11 & 0.15 & 0.30 & 2.57 & 0.11 \\
\hline $\begin{array}{l}\text { Geosonda } \\
\text { Konsolidacija, Beograd }\end{array}$ & 1.00 & 0.39 & 0.21 & 0.92 & 2.01 & 0.39 \\
\hline Graditelj, Beograd & 1.00 & 0.03 & 0.03 & 0.62 & 1.84 & 0.03 \\
\hline Termika, Beograd & 0.00 & 4.55 & 4.59 & 0.94 & 1.06 & 0.00 \\
\hline $\begin{array}{l}\text { Hidrotehnika } \\
\text {-hidroenergetika, } \\
\text { Beograd } \\
\end{array}$ & 1.00 & 0.13 & 0.08 & 0.55 & 3.01 & 0.13 \\
\hline $\begin{array}{l}\text { Zlatibor gradnja, } \\
\text { Beograd }\end{array}$ & 0.50 & 7.94 & 4.65 & 1.06 & 1.61 & 3.97 \\
\hline Izoprogres, Beograd & 1.00 & 11.62 & 32.34 & 0.33 & 1.10 & 11.62 \\
\hline Jedinstvo, Sevojno & 0.64 & 11.20 & 5.98 & 0.98 & 1.90 & 7.17 \\
\hline Napredak, Pirot & 1.00 & 0.09 & 0.07 & 1.10 & 1.31 & 0.09 \\
\hline $\begin{array}{l}\text { Novi Pazar put, Novi } \\
\text { Pazar }\end{array}$ & 1.00 & 5.54 & 4.35 & 0.78 & 1.63 & 5.54 \\
\hline Planum, Beograd & 0.98 & 2.53 & 0.80 & 0.92 & 3.43 & 2.48 \\
\hline Putevi, Čačak & 1.00 & 7.92 & 4.86 & 0.52 & 3.16 & 7.92 \\
\hline Putevi, Užice & 1.00 & 7.88 & 0.55 & 2.41 & 5.93 & 7.88 \\
\hline $\begin{array}{l}\text { PZP Požarevac, } \\
\text { Požarevac }\end{array}$ & 1.00 & 1.07 & 0.68 & 0.90 & 1.74 & 1.07 \\
\hline Srbijaput, Beograd & 1.00 & 3.39 & 0.37 & 1.82 & 5.06 & 3.39 \\
\hline Sremput, Ruma & 1.00 & 0.24 & 0.22 & 0.40 & 2.68 & 0.24 \\
\hline Standard, Leskovac & 1.00 & 0.52 & 0.86 & 0.47 & 1.30 & 0.52 \\
\hline
\end{tabular}

Source: Representation by the authors based on data from www.belex.rs

In 2012 six companies paidout dividends, where one company, Planum, paidout dividends in the amount of net profit and thus recorded zero sustainable growth rate. In 2013, in the sector of construction companies there was introduced stricter dividend policy and only four companies paid out dividends from the profit.

The highest rate of return on equity in 2012 was achieved in construction company Zlatibor gradnja - Belgrade with 40.02\%, and in 2013 Energoprojekt oprema - Belgrade with $21.06 \%$. The highest rate of profit margins in 2012 and 2013 had the company Izoprogres - Belgrade, 25.98\% in 2012 and 32.34\% in 2013. The ratio of total asset turnover of construction enterprises decreased and its average was 1.17 in 2012 and 0.91 in 2013. The highest use efficiency of total assets was reported in the enterprises Energoprojekt oprema 2.72 in 2012 and Putevi Užice 2.41 in 2013. Own capital multiplier of construction enterprises ranges from 1.06 to 9.58 , wherein this indicator for a larger number of construction enterprises is at a level of about 2 and over 3 , ie. at a much higher level than in the agricultural and food enterprises. In both years, the 
highest level of own capital multiplier was recorded in the enterprise Putevi Užice 9.58 in 2012 and 5.93 in 2013.

Generally, the high level of own capital multiplier indicates that these enterprises are considerably more reliant on borrowed sources of financing than the sampled agricultural and food enterprises, namely they have significantly higher level of indebtedness compared to analyzed agricultural and food enterprises, which is in line with the results of existing research (Racic et al., 2011).

\section{Comparative analysis of sustainable growth rate of enterprises in Serbia}

After establishing a sustainable growth rate of agricultural, food and construction enterprises, the question is whether there is a significant difference among them and what is the relationship between sustainable growth rate and the inflation rate in order to establish the real growth potential. The average value of sustainable growth rate of enterprises in the sample of different sectors is shown in Table 7.

Table 7. Descriptive statistics of sustainable growth rate values of sampled enterprises

\begin{tabular}{|l|r|r|r|r|r|r|}
\hline & $\begin{array}{c}\text { Agriculture } \\
\mathbf{2 0 1 2}\end{array}$ & $\begin{array}{c}\text { Agriculture } \\
\mathbf{2 0 1 3}\end{array}$ & $\begin{array}{c}\text { Food industry } \\
\mathbf{2 0 1 2}\end{array}$ & $\begin{array}{c}\text { Food } \\
\text { industry } \\
\mathbf{2 0 1 3}\end{array}$ & $\begin{array}{c}\text { Constr } \\
\text { uction } \\
\mathbf{2 0 1 2}\end{array}$ & $\begin{array}{c}\text { Construction } \\
\mathbf{2 0 1 3}\end{array}$ \\
\hline \multirow{2}{*}{$\mathrm{N}$ Valid } & 20 & 20 & 20 & 20 & 20 & 20 \\
\cline { 2 - 7 } Missing & 0 & 0 & 0 & 0 & 0 & 4.192 \\
\hline Mean & 9.406 & 5.775 & 7.861 & 9.8705 & 8.2665 & 1.795 \\
\hline Median & 6.175 & 3.435 & 7.345 & 7.695 & 3.305 & 5.39729 \\
\hline $\begin{array}{l}\text { Std. } \\
\text { Deviation }\end{array}$ & 10.84075 & 9.34073 & 10.53863 & 8.55146 & 10.92532 & 1.815 \\
\hline Skewness & 2.206 & 0.915 & -0.706 & 1.088 & 1.466 & 0.512 \\
\hline $\begin{array}{l}\text { Std. Error of } \\
\text { Skewness }\end{array}$ & 0.512 & 0.512 & 0.512 & 0.512 & 0.512 & 3.894 \\
\hline Kurtosis & 6.181 & 3.334 & 2.634 & 0.29 & 1.033 & 0.992 \\
\hline $\begin{array}{l}\text { Std. Error of } \\
\text { Kurtosis }\end{array}$ & 0.992 & 0.992 & 0.992 & 0.992 & 0.992 & 0 \\
\hline Minimum & 0.63 & -14.74 & -21.95 & 0.6 & 0 & 21.06 \\
\hline Maximum & 45.97 & 32.46 & 28.44 & 28.97 & 36.42 & \\
\hline
\end{tabular}

Source: Representation by the authors based on SPSS

Using comparative analysis it can be identified that in 2012, enterprises from agricultural sector had the highest sustainable growth rate $(9.4060 \%)$, while in 2013 those were food enterprises (9.8705\%). In 2012 enterprises from food sector had the lowest sustainable growth rate $(7.8610 \%)$, and in 2013 construction sector enterprises $(4.1920 \%)$. The maximum deviation from the average values of the individual sustainable growth rate is recorded in construction enterprises in 2012, while in 2013 it was recorded in agricultural enterprises. Small deviations of the average sustainable 
growth rate between the food enterprises can be noticed from year 2012 to year 2013, while in the agricultural and construction enterprises this deviation is higher than in the previous year.

The normality test of sustainable growth rate distribution showed that the hypothesis of normal sustainable growth rate distribution can not be accepted for enterprises from the construction and the agricultural sector in 2012, and for all three sectors' enterprises in 2013 (Shapiro-Wilk, Sig. <0.05) (Table 8).

Table 8. Testing normality of distribution of sustainable growth rate

\begin{tabular}{|l|r|r|r|r|r|r|r|}
\hline \multicolumn{9}{|c|}{ Tests of Normality } \\
\hline \multirow{3}{*}{ Activity } & \multicolumn{2}{|c|}{ Kolmogorov-Smirnov } & \multicolumn{2}{|c|}{ Shapiro-Wilk } \\
\cline { 2 - 9 } & & Statistic & \multicolumn{1}{c|}{ df } & \multicolumn{1}{c|}{ Sig. } & Statistic & \multicolumn{1}{c|}{ df } & \multicolumn{1}{c|}{ Sig. } \\
\hline \multirow{3}{*}{$\begin{array}{l}\text { Sustainable growth rate } \\
2012\end{array}$} & 1 & 0.209 & 20 & 0.022 & 0.76 & 20 & 0 \\
\cline { 2 - 9 } & 2 & 0.184 & 20 & 0.074 & 0.927 & 20 & 0.138 \\
\cline { 2 - 9 } & 3 & 0.278 & 20 & 0 & 0.746 & 20 & 0 \\
\hline \multirow{3}{*}{$\begin{array}{l}\text { Sustainable growth rate } \\
2013\end{array}$} & 1 & 0.228 & 20 & 0.008 & 0.87 & 20 & 0.012 \\
\cline { 2 - 9 } & 2 & 0.19 & 20 & 0.057 & 0.869 & 20 & 0.011 \\
\cline { 2 - 9 } & 3 & 0.219 & 20 & 0.013 & 0.778 & 20 & 0 \\
\hline
\end{tabular}

Source: Representation by the authors based on SPSS

Hence, the Kruskal-Wallis-test was used in order to establish existance of statistically significant differences in the level of sustainable growth rate of observed sector enterprises. Results of testing hypotheses 2 and 3 based on the Kruskal-Wallis-test showed that there is no statistically significant difference among sustainable growth rates of enterprises from the listed sectors $\left(c^{2}(2, n=60)=1.527, p=0.466\right.$ for 2012 and $\mathrm{c}^{2}(2, \mathrm{n}=60)=8.181, \mathrm{p}=0.017$ for 2013). On this basis, it can be concluded that the differences in the sustainable growth rate of enterprises from different sectors are random and that the medians in 2012 are equal for agriculture and food sectors, while they are different in all three sets (strata) in 2013 (Table 9). 
Table 9. Results of the Kruskal-Wallis - test for 2012 and 2013

\begin{tabular}{|c|c|c|c|c|}
\hline \multicolumn{5}{|c|}{ Test Statistics ${ }^{\mathrm{a}, \mathrm{b}}$} \\
\hline & & Sustainable grow. rate 2012 & \multicolumn{2}{|c|}{ Sustainable grow. rate 2013} \\
\hline \multicolumn{2}{|l|}{ Chi-Square } & 1.527 & \multicolumn{2}{|r|}{8.181} \\
\hline \multicolumn{2}{|l|}{ df } & 2 & \multicolumn{2}{|r|}{2} \\
\hline \multicolumn{2}{|l|}{ Asymp. Sig. } & .466 & \multicolumn{2}{|r|}{.017} \\
\hline \multicolumn{5}{|c|}{$\begin{array}{l}\text { a. Kruskal Wallis Test } \\
\text { b. Grouping Variable: Ac }\end{array}$} \\
\hline \multicolumn{5}{|c|}{ Frequencies } \\
\hline & & \multicolumn{3}{|c|}{ Activity } \\
\hline & & $1=$ agricult. & $2=$ food & $3=$ construc. \\
\hline \multirow{2}{*}{$\begin{array}{l}\text { Sustainable grow. } \\
\text { rate2012 }\end{array}$} & $>$ Median & 11 & 11 & 8 \\
\hline & $<=$ Median & 9 & 9 & 12 \\
\hline \multirow{2}{*}{$\begin{array}{l}\text { Sustainable grow. } \\
\text { rate } 2013\end{array}$} & $>$ Median & 9 & 13 & 8 \\
\hline & $<=$ Median & 11 & 7 & 12 \\
\hline
\end{tabular}

Source: Representation by the authors based on SPSS

For the evaluation of statistically significant difference in the level of sustainable growth rate of a sector and sustainable growth rates of all observed enterprises in 2013 compared to 2012 , for testing hypotheses 4, Wilcoxon Signed Rank was used (Table 10).

Table 10. Results of the Wilcoxon Signed Rank test for 2012 and 2013

\begin{tabular}{|c|c|c|c|c|}
\hline \multicolumn{5}{|c|}{ Test Statistics ${ }^{b}$} \\
\hline & $\begin{array}{l}\text { Agriculture_2013- } \\
\text { Agriculture_2012 }\end{array}$ & $\begin{array}{l}\text { Food_2013- } \\
\text { Food_2012 }\end{array}$ & $\begin{array}{c}\text { Construc._2013 } \\
- \\
\text { Construc._2012 }\end{array}$ & $\begin{array}{c}\text { Sustainable } \\
\text { growth r._2013- } \\
\text { Sustainable } \\
\text { growth r.- 2012 }\end{array}$ \\
\hline Z & $-1.630^{\mathrm{b}}$ & $-.336^{\mathrm{a}}$ & $-2.012^{\mathrm{a}}$ & $-2.276^{\mathrm{a}}$ \\
\hline $\begin{array}{l}\text { Asymp. Sig. } \\
\text { (2-tailed) }\end{array}$ & 0.103 & 0.737 & .044 & .023 \\
\hline \multicolumn{4}{|c|}{$\begin{array}{l}\text { a. Based on positive ranks. } \\
\text { b. Based on negative ranks. } \\
\text { c. Wilcoxon Signed Ranks Test }\end{array}$} & \\
\hline
\end{tabular}

Source: Representation by the authors based on SPSS

Results of Wilcoxon Signed Rank test for 2012 and 2013 showed there is statistically significant difference in the overall level of sustainable growth rates in the observed years $(z=-2.276$, sig $=0.023)$, but that there is no statistically significant difference in the level of sustainable growth rates of agricultural enterprises $(z=-1.630$, sig $=0.103)$ and food enterprises $(z=-0.336$, sig $=0.737)$, while there is statistically significant difference in construction enterprises $(z=-2.012$, sig $=0,044)$ in 2013 compared to 2012, thus hypothesis 4 is partially verified and partially rejected.

For the purposes of making a valid conclusion about the real growth potential of sampled agricultural, food and construction enterprises, the inflation rate is included in 
the analysis as well. Results of testing hypotheses 5 and 6 are given in Table 11 .

Table 11. Results One Sample T test for 2012 and 2013

\begin{tabular}{|c|c|c|c|c|c|c|c|}
\hline \multicolumn{8}{|c|}{ One-Sample Statistics } \\
\hline & & $\mathbf{N}$ & Mean & \multicolumn{2}{|c|}{ Std. Deviation } & \multicolumn{2}{|c|}{$\begin{array}{l}\text { Std. Error } \\
\text { Mean }\end{array}$} \\
\hline \multicolumn{2}{|c|}{ Sustainable growth r._2012 } & 60 & 8.5112 & \multicolumn{2}{|c|}{10.60594} & \multicolumn{2}{|r|}{1.36922} \\
\hline \multicolumn{2}{|c|}{ Sustainable growth r._2013 } & 60 & 6.6125 & \multicolumn{2}{|c|}{8.17617} & \multicolumn{2}{|r|}{1.05554} \\
\hline \multicolumn{8}{|c|}{ One-Sample Test } \\
\hline & \multicolumn{7}{|c|}{ Test Value $=12.2(2012$.$) ; Test Value =2.2(2013)}$. \\
\hline & \multirow[t]{2}{*}{$\mathbf{t}$} & \multirow[t]{2}{*}{ df } & \multirow[t]{2}{*}{$\begin{array}{c}\text { Sig. } \\
\text { (2-tailed) }\end{array}$} & \multirow[t]{2}{*}{$\begin{array}{c}\text { Mean } \\
\text { Difference }\end{array}$} & \multicolumn{3}{|c|}{$\begin{array}{l}95 \% \text { Confidence } \\
\text { Interval of the } \\
\text { Difference }\end{array}$} \\
\hline & & & & & Lowe & & Upper \\
\hline $\begin{array}{l}\text { Sustainable growth } \\
\text { r._2012 }\end{array}$ & -2.694 & 59 & .009 & -3.68883 & & & -.9490 \\
\hline $\begin{array}{l}\text { Sustainable growth } \\
\text { r._2013 }\end{array}$ & 4.180 & 59 & .000 & 4.41250 & & & 6.5246 \\
\hline
\end{tabular}

Source: Representation by the authors based on SPSS

It can be concluded that there is a statistically significant difference between the average sustained growth rate of sampled enterprises (Mean $=8.5112 \%$, St.Dev $=$ 10.60594) and inflation rate measured by the consumer price index in 2012 (12.2\%). The hypothesis 5 is rejected and it can be considered that the average sustained growth rate of the company in 2012 is lower than the inflation rate measured by the consumer price index. Furthermore, given that the results of testing hypotheses 2 showed no statistically significant differences among the sustainable growth rate of agricultural, food and construction enterprises in 2012, it can be concluded that the average sustainable growth rates of enterprises from these sectors are lower than the inflation rate. Namely, as achieved $\mathrm{t}$-value of -2.694 is less than the limited tabular value $(\mathrm{t}=$ 2.00), for 59 degrees of freedom and materiality threshold of $p=0.05$, the hypothesis 5 is rejected and alternative hypothesis with error $\mathrm{p}<0.05$ and certainty $\mathrm{P}>95 \%$ claiming that in the 2012 the average sustainable growth rate is lower than the inflation rate is accepted, that leads to the conclusion that in this year there was no considerable potential for growth of sampled enterprises.

The hypothesis 6 is also rejected as the average sustainable growth rate of enterprises $($ Mean $=6.6125 \%$, St.Dev $=8.17617)$ in 2013 was statistically significantly different than the inflation rate measured by the consumer price index $(2.2 \%)$. The results indicate that the average sustainable growth rate was significantly higher than the inflation rate. Based on the results of testing hypotheses 3 which indicated that there were no statistically significant differences among the sustainable growth rate of agricultural, food and construction enterprises in 2013 and hypotheses 6, it can be concluded that the average sustainable growth rates of enterprises in the agricultural, food and construction sectors are higher than the inflation rate. Namely, as achieved t-value of 
4.180 is higher than the limited tabular value $(t=2.00)$, for 59 degrees of freedom and level signification of $\mathrm{p}=0.05$, the hypothesis 6 is rejected and alternative hypothesis with error $\mathrm{p}<0.05$ and safety $\mathrm{P}>95 \%$ claiming that in 2013 the average sustainable growth rate is higher than the inflation rate is accepted, that leads to the conclusion that in this year there was a considerable potential for growth in sampled food, agricultural and construction enterprises.

\section{Conclusion}

Thanks to its geographical location, natural characteristics of the soil and favorable climatic conditions, agricultural production in Serbia has great potential for development. Therefore, it could be expected that enterprises from the agricultural sector as well as food enterprises have a high sustainable growth rate. This study showed that there is no statistically significant differences among the sustainable growth rate of agricultural, food and construction sectors in 2012 and 2013. Hence it can be concluded that Serbia does not sufficiently use all the competitive advantages it has in agriculture and food industry.

Research results show that there is no statistically significant difference in the level of sustainable growth rates of sampled enetrprises, but that there is statistically significant difference in the overall level of sustainable growth rates in 2013 compared to 2012. Research indicates that there is no statistically significant difference in the level of sustainable growth rates of agricultural and food enterprises in 2013 compared to 2012, which further indicates that in 2013 compared to 2012 there were no significant changes in the level of potential for the development of these sectors. In the control sector in this research, in construction sector enterprises, there is statistically significant difference in the level of sustainable growth rate in 2013 compared to 2012, ie. there was significant deterioration in the development potential of this sector. This points out to better vitality and greater development potential of the agricultural and food sectors.

Also, it was found that the average sustainnable growth rate of enterprises in 2012 was lower than the inflation rate measured by the consumer price index, so it can be concluded that in this year there was no real potential for growth of these enterprises. In 2013, there were no significant changes in the level of the average sustainable growth rate compared to the year 2012, but there was significant decline in the inflation rate comparing to the previous year. It can be concluded that for the real growth potential of agricultural and food enterprises as well as enterprises in other sectors, it is necessary to have macroeconomic price stability, which in some years may prevaile over the microeconomic indicators of particular business enterprises and branches.

The study results lead to the conclusion that under the assumption of agricultural and food enterprises growth at the level of sustainable growth rate, satisfactory real growth of named sectors is achieved only in cases of macroeconomic price stability and more extensive and diversified credit support. The research can serve as a basis for further research on this topic in the direction of the comparative analysis of particular 
enterprises and particular branches of activity within the agricultural sector in order to determine those agricultural industries that in Serbia have the greatest development potential measured by the sustainable growth rate.

\section{Literature}

1. Ashta, A. (2008): Sustainable Growth Rates: Refining a Measure. Strategic Change, No. 17, pp. 207-214.

2. Brealey, R., Myers, S. (2003): Principles of Corporate Finance (7th ed.). New York: McGraw-Hill.

3. Chang, Y.C. (2012): Strategy formulation implications from using a sustainable growth model, Journal of Air Transport Management, No. 20, pp. 1-3.

4. Chen, H.Y., Gupta, M.C., Lee, A.C., Lee, C.F. (2013): Sustainable growth rate, optimal growth rate, and optimal payout ratio: A joint optimization approach, Journal of Banking \& Finance, Vol. 37, No. 4, pp. 1205-1222.

5. Clouse, M., McFaddin, S. (1994): Sustainable growth in the US gas utility industry, Resources Policy, Vol. 20, No. 2, pp. 117-123.

6. Fonseka, M.M., Ramos, C.G., Tian, G. (2012): The Most Appropriate Sustainable Growth Rate Model For Managers And Researchers, The Journal of Applied Business Research, Vol. 28, No. 3, pp. 481-500.

7. Fuller, R., Hsia, C.C.(1984): A Simplified Common Stock Valuation Model, Financial Analysts Journal, Vol. 40. No. 5, pp. 49-56.

8. Gordon, M. (1959): Dividends, Earnings and Stock Prices, Review of Economics and Statistics, Vol. 41, No. 2, pp. 99-105.

9. Higgins, R.C.(1977): How Much Growth Can a Firm Afford? Financial Management, Vol. 6, No. 3, pp. 7-16.

10.Higgins, R.C. (1981): Sustainable Growth Under Inflation. Financial Management, Vol. 10, No. 4, pp. 36-40.

11. Ministarstvo finansija (2014): Osnovni indikatori makroekonomskih kretanja, (available at: http://www.mfin.gov.rs).

12.Pinto, J., Henry, E., Robinson R.T., Stowe D.J. (2010): Equitu Asset Valuation, Hoboken, New Jersey, John Wiley \& Sons, Inc.

13.Račić, Ž., Barjaktarović, L., Zeremski, A. (2011): Analiza uticaja zaduženosti na profitabilnost uspešnih domaćih kompanija u uslovima ekonomske krize, Industrija, Vol. 39, No. 3, pp. 45-60.

14.Seens, L.D. (2013): Small and Medium-Sized Enterprises Growth Study: Actual vs. Sustainable Growth, Canada, Small Business Branch Research and Analysis Directorate.

15.Stowe, J. (2000): Analysis of Equity Investments. Boston: AIMR. Pearson Custom Publishin.

16.Van Horne, J.C., Wachowicz, J.M. (2007): Osnovi finansijskog menadžmenta. 
Beograd: Datastatus.

17.Virani, V. (2013): Sustainable Growth Rate: Refining a Measure - a Case Study of Tata Motors and Maruti Suzuki, Indian Journal of Applied Research, Vol. 3, No. 4, pp. 317-319.

Websites:

1. Official Website of the Belgrade Stock Exchange, http://www.belex.rs/ (retrived 20.05.2015.)

2. Official Website of the Agency for Business Registers, www.apr.gov.rs (retrived 22.05. 2015.) 


\title{
ODRŽIVA STOPA RASTA POLJOPRIVREDNIH I PREHRAMBENIH PREDUZEĆA U SRBIJI
}

\author{
Zoran Jović5 , Kosana Vićentijevič ${ }^{6}$, Nataša Glišović ${ }^{7}$ \\ Rezime
}

Mnoga profitabilna preduzeća mogu otići u bankrotstvo ukoliko rastu presporo ili rastu prebrzo. Da bi se ocenilo da li neko preduzeće raste presporo ili prebrzo, potrebno je utvrditi njegovu održivu stopu rasta, koja predstavlja maksimalnu stopu rasta koje preduzeće može ostvariti bez dodatnog zaduživanja. Cilj ovog istraživanja je da se utvrdi održiva stopa rasta za preduzeća poljoprivrednog i prehrambenog sektora u Srbiji $u$ 2012. i 2013. godini i da se utvrdi da li postoje razlike između ova dva međusobno povezana sektora. U istraživanje je uveden i građevinski sektor kao kontrolni sektor koji reprodukciono nije povezan sa poljoprivredom i prehranom. Takođe, cilj je da se utvrdi realna održiva stopa rasta posmatranih sektora u Srbiji uzimanjem u obzir stope inflacije koja u Srbiji ima vidljive oscilacije u posmatranim godinama. Realna održiva stopa rasta služi kao orijentir za donošenje zaključaka o razvojnom potencijalu ovih grana i zaključivanju koji su interni ili eksterni podsticaji potrebni za dalje povećanje održive stope rasta, odnosno potencijala za rast. Limitiranost obima rada je usmerila istraživanje na utvrđivanje održive stope rasta i međusobnu komparaciju posmatranih sektora i dala osnov za dalja istraživanja u pravcu komparativne analize unutar poljoprivrednog sektora $i$ određivanja poljoprivrednih delatnosti sa najvećim razvojnim potencijalom izraženim kroz održivu stopu rasta.

Ključne reči: održiva stopa rasta, poljoprivredni sektor, prehrambeni sektor, politika isplate dividendi

5 Vanredni profesor, dr Zoran Jović, vanredni profesor, Univerzitet Singidunum, Poslovni fakultet u Valjevu, Železnička 5, 14000 Valjevo, Srbija, Telefon: +381 14292 610, E-mail: zjovic@singidunum.ac.rs

6 Vanredni profesor, dr Kosana Vićentijević, vanredni profesor, Univerzitet Singidunum Beograd, Danijelova 32, 11000 Beograd, Srbija, Telefon: +381 113093 209, E-mail: kvicentijevic@singidunum.ac.rs

7 Master Nataša Glišović, Matematički institut Srpske akademije nauka i umetnosti (SANU), 11000 Beograd, Ulica Kneza Mihaila br. 36, Telefon: + 381603630 170, E-mail: natasaglisovic@gmail.com 